\title{
INVARIANT APPROACHES TO EQUATIONS OF FINANCE
}

\author{
F. M. Mahomed ${ }^{1}$, K. S. Mahomed ${ }^{1}$, R. Naz $^{2}$ and E. Momoniat ${ }^{1}$ \\ ${ }^{1}$ Differential Equations, Continuum Mechanics and Applications, \\ School of Computational and Applied Mathematics, University of the \\ Witwatersrand,Private Bag 3, Wits 2050, South Africa \\ ${ }^{2}$ Centre for Mathematics and Statistical Sciences,Lahore School of Economics, Lahore, \\ 53200, Pakistan \\ Fazal.Mahomed@wits.ac.za
}

\begin{abstract}
We firstly show how effective it is to utilize the invariant criteria for scalar linear $(1+1)$ parabolic equations in order to perform reductions to the three Lie canonical forms of a bond-pricing model from finance. As a consequence we arrive at new results on bond-pricing equations that admit four nontrivial symmetries. In the second part we draw attention to a new method developed for equations of economics.
\end{abstract}

\section{INTRODUCTION}

Many contributions in the utility of Lie symmetries and operator approaches to differential equations have appeared in the literature since the earliest works of Lie on the subject.

Recent studies have utilized Lie symmetries for many partial differential equations (PDEs) of finance. One of the initial investigations is due to Gazizov and Ibragimov [1] who uncovered the algebraic properties of the Black-Scholes equation. Goard [2] obtained solutions to bond-pricing equations by use of the classical Lie method. Lo and Hui [3] invoked the algebraic method to value financial derivatives. Fundamental solutions to zero-coupon bond pricing equations were deduced by Pooe et al. [4]. More recently there have been a spate of papers (see, e.g. Sophocleous et al. [5] and refs. theirin). This includes nonlinear equations of finance such as the Stein-Stein model.

One of the earlier studies that proposed the invariant approach to Fokker-Planck equations was initiated by Johnpillai and Mahomed [6]. They [6] proved the necessary and sufficient condition for a scalar linear $(1+1)$ parabolic equation, in terms of the coefficient of the equation, to be reducible via equivalence transformations to the classical heat equation. Inter alia they showed how the Black-Scholes equation can be transformed to the classical heat equation. In later work, Mahomed [7] presented a complete invariant characterization of scalar linear (1+1) parabolic PDE of one space variable and one time variable

$$
u_{t}=a(t, x) u_{x x}+b(t, x) u_{x}+c(t, x) u
$$

where $a, b$ and $c$ are continuous differentiable functions of $t$ and $x$. Lie [8] provided the complete group classification of the parabolic PDE (1) for which (1) has nontrivial point symmetry algebras of dimensions 1, 2, 4 and 6 apart from the infinite dimensional algebra of trivial Lie symmetries of the superposition operators.

In the first part of the paper, we review the invariant conditions found in [7]. We 
show how these invariant conditions can elegantly be used to classify an important class of bond pricing equations from finance for which a symmetry group classification attempt was performed in Sinkala et al. [9]. Here we demonstrate how this can be achieved in a simpler way using an invariant approach. W also find new cases for which the bond pricing equations considered admit four nontrivial symmetries and hence can be transformed to the second Lie canonical form.

In the second part of the paper we draw attention to the new Hamiltonian approach to equations of economics. There is a paucity of exact solutions for equations of economic theory. Qualitative and quantitative methods are mainly invoked (see, e.g. Ruiz-Tamarit and Venturo-Marco [10]). Exact solutions to dynamic economic models are due to ad-hoc techniques (see, e.g. Chilarescu [11]). A new method [12] has been developed within a Hamiltonian framework for many state, costate and control variables. This approach gives rise to first integrals, reductions and even solutions to these dynamical equations of economics.

The layout of the paper is as follows. In the next section we briefly capture the salient features of the invariant criteria for scalar linear $(1+1)$ parabolic PDEs as proved in [7]. Then we apply this to bond pricing equations of finance taken from [9]. Section 3 is on the Hamiltonian method of equations of economics. A concise conclusion is presented at the end.

\section{APPLICATION OF THE INVARIANT METHOD TO FINANCE}

We firstly review the main results of [7] on invariant criteria for scalar linear parabolic PDEs (1).

Equivalence transformations [8] of the parabolic PDE (1) comprise linear change of the dependent variable

$$
\bar{u}=\sigma(t, x) u, \quad \sigma \neq 0
$$

and transformations of the independent variables

$$
\bar{t}=\phi(t), \quad \bar{x}=\psi(t, x), \quad \dot{\phi} \neq 0, \psi_{x} \neq 0,
$$

in which $\phi, \psi$ and $\sigma$ are arbitrary functions with restrictions for invertibility of the transformations.

Two linear parabolic PDEs of the form (1) are called equivalent to each other if one can be transformed to the other by the equivalence transformations (2) and (3).

Lie [8] showed that a scalar linear parabolic PDE (1) has the following four canonical forms.

$$
\begin{aligned}
& u_{t}=u_{x x}, \\
& u_{t}=u_{x x}+A / x^{2} u, A \neq 0 \\
& u_{t}=u_{x x}+c(x) u, c \neq 0, A / x^{2}, \\
& u_{t}=u_{x x}+c(t, x) u, c \neq 0, A / x^{2} .
\end{aligned}
$$

The heat equation, which is the first Lie canonical form, has six nontrivial symmetries apart from the infinite number of trivial superposition symmetries. The second Lie canonical form as given above in (4) has four nontrivial symmetries. The third in general has two symmetries and the fourth has in general one nontrivial symmetry. 
We now state the following theorem which provides a practical test for reduction to the Lie canonical forms.

Theorem (see [7]). The scalar linear (1+1) parabolic PDE (1) is reducible via equivalence transformations (2) and (3) to

(a) the heat equation

$$
\bar{u}_{\bar{t}}=\bar{u}_{\bar{x} \bar{x}}
$$

if and only if the coefficients of the parabolic PDE (1) satisfies the invariant condition

$$
2 L_{x}+2 M_{x}-N_{x}=0
$$

where

$$
L=|a|^{1 / 2}\left[|a|^{1 / 2} J_{x}\right]_{x}, M=|a|^{1 / 2}\left[|a|^{1 / 2} \partial_{t}(b /(2 a))\right]_{x}, N=|a|^{1 / 2} \partial_{t}^{2}\left(1 /|a|^{1 / 2}\right),(7)
$$

and $J$ is

$$
J=c-\frac{b_{x}}{2}+\frac{b a_{x}}{2 a}+\frac{a_{x x}}{4}-\frac{3}{16} \frac{a_{x}^{2}}{a}-\frac{a_{t}}{2 a}-\frac{b^{2}}{4 a}
$$

(b) the second Lie canonical equation

$$
\bar{u}_{\bar{t}}=\bar{u}_{\bar{x} \bar{x}}+A / \bar{x}^{2} \bar{u}
$$

where $A$ is a nonzero constant, if and only if the coefficients of parabolic PDE (1) satisfy the invariant equation, provided that condition (6) does not hold,

$$
\begin{aligned}
& 20 L_{x}+20 M_{x}-10 N_{x}+10\left[|a|^{1 / 2} M_{x}\right]_{x} \int d x /|a|^{1 / 2}-5\left[|a|^{1 / 2} N_{x}\right]_{x} \int d x /|a|^{1 / 2} \\
& +10\left[|a|^{1 / 2} L_{x}\right]_{x} \int d x /|a|^{1 / 2}+\left[|a|^{1 / 2}\left[|a|^{1 / 2} L_{x}\right]_{x}\right]_{x}\left(\int d x /|a|^{1 / 2}\right)^{2} \\
& +\left[|a|^{1 / 2}\left[|a|^{1 / 2} M_{x}\right]_{x}\right]_{x}\left(\int d x /|a|^{1 / 2}\right)^{2}-\frac{1}{2}\left[|a|^{1 / 2}\left[|a|^{1 / 2} N_{x}\right]_{x}\right]_{x}\left(\int d x /|a|^{1 / 2}\right)^{2}=0,
\end{aligned}
$$

where $L, M, N$ and $J$ are as given in (7) and (8);

(c) the third Lie canonical form

$$
\bar{u}_{\bar{t}}=\bar{u}_{\bar{x} \bar{x}}+\bar{c}(\bar{x}) \bar{u}
$$

with two nontrivial symmetries if and only if the coefficients of parabolic equation (1) satisfy the invariant criterion, provided that the conditions (6) and (10) do not apply,

$$
\frac{\partial}{\partial t}\left(J+\partial_{t} \int \frac{b}{2 a} d x-\frac{1}{2} \int 1 /|a|^{1 / 2}\left(\partial_{t}^{2} \int d x /|a|^{1 / 2}\right) d x\right)=0
$$

(d) the fourth Lie canonical form

$$
\bar{u}_{\bar{t}}=\bar{u}_{\bar{x} \bar{x}}+\bar{c}(\bar{t}, \bar{x}) \bar{u}
$$

having one nontrivial symmetry if and only if the coefficients of parabolic PDE (1) satisfy the condition, provided that the conditions (6) and (10) do not hold,

$$
\frac{\partial}{\partial t}\left(J+\partial_{t} \int \frac{b}{2 a} d x-\frac{1}{2} \int 1 /|a|^{1 / 2}\left(\partial_{t}^{2} \int d x /|a|^{1 / 2}\right) d x\right) \neq 0 .
$$
from [9].

We apply the above Theorem to a class of bond pricing equations of finance taken The symmetry group classification of the bond pricing PDE 


$$
u_{t}=-\frac{1}{2} \rho^{2} x^{2 \gamma} u_{x x}-\left(\alpha+\beta x-\lambda \rho x^{\gamma}\right) u_{x}+x u,
$$

where $\alpha, \beta, \gamma, \rho$ and $\lambda$ are constants, was attempted in [9]. Also the motivation for the symmetry study was made clear as (15) contains the Vasicek [13] and Cox-Ingersoll-Ross (CIR) [14] models which correspond to $\lambda=0$ and $\gamma=1 / 2$ respectively.

In [9], the authors investigated (15) for possible extensions of the principal Lie algebra for various values of the parameter $\lambda$. They found this to be the case for $\lambda=0,1 / 2,3 / 2,2$.

We use the invariant approach encapsulated in the Theorem above to simply and elegantly find all values of $\lambda$ for which the PDE (15) can be mapped to the Lie canonical forms. We discover new cases for $\lambda=2,3 / 2$ and $\lambda=1 / 2$ which were previously not reported in [9] or in the literature.

It is easy to evaluate $J$ as in (8) for the bond pricing PDE (15). Indeed it is

$$
\begin{aligned}
& J=x+\frac{1}{2} \beta-\beta \gamma+\frac{1}{2} \lambda^{2}+\frac{1}{2} \lambda \rho \gamma x^{\gamma-1}-\frac{1}{8} \rho^{2} \gamma^{2} x^{2 \gamma-2}+\frac{1}{4} \rho^{2} \gamma x^{2 \gamma-2}-\alpha \gamma x^{-1} \\
& +\frac{1}{2} \frac{\alpha^{2}}{\rho^{2}} x^{-2 \gamma}+\frac{\alpha \beta}{\rho^{2}} x^{1-2 \gamma}-\frac{\alpha \lambda}{\rho} x^{-\gamma}-\frac{\beta \lambda}{\rho} x^{1-\gamma}+\frac{\beta^{2}}{2 \rho^{2}} x^{2-2 \gamma} .
\end{aligned}
$$

We first look at the reduction to the heat equation. Since the PDE (15) is autonomous, the invariant condition (6) for reduction to the heat equation becomes $L_{x}=0$ and as a consequence we have that (16) when evaluated for the coefficients of the PDE (15) gives

$$
\begin{aligned}
& \frac{1}{2} \rho^{2} \gamma(2 \gamma-1) x^{2 \gamma-2}+\frac{3}{2} \lambda \rho^{3} \gamma(\gamma-1)^{3} x^{3 \gamma-4}-\frac{3}{2} \rho^{4} \gamma^{2}(\gamma-1)^{3} x^{4 \gamma-5} \\
& +3 \rho^{4} \gamma(\gamma-1)^{3} x^{4 \gamma-5}+\frac{1}{2} \alpha \rho^{2} \gamma(\gamma-2)(2 \gamma-3) x^{2 \gamma-4}-\alpha^{2} \gamma(\gamma+1) x^{-3} \\
& +\frac{1}{2} \alpha \beta(1-2 \gamma) \gamma x^{-2}-\frac{1}{2} \alpha \lambda \rho \gamma(\gamma-2) x^{\gamma-3}=0 .
\end{aligned}
$$

Quite clearly all powers of $x$ in (17) cannot be distinct otherwise we arrive at $\rho=0$ which is inadmissible. Therefore we need to consider values of the parameters for which the powers of $x$ are the same. As an example, if $2 \gamma-2=3 \gamma-4$, we immediately have $\gamma=2$ and the equating of separate powers of $x$ to zero in (17) quickly yields $\alpha=0$ and $\lambda=-1 / \rho$. This corresponds to the case found in [9]. In a similar manner we determine the other nontrivial cases for which the invariant condition(17) holds. We summarize all our findings below.

I. $\gamma=2, \alpha=0, \lambda=-1 / \rho, \beta$ and $\rho$ are arbitrary

II. $\gamma=0, \alpha, \beta, \lambda$ and $\rho$ are arbitrary

III. $\gamma=1 / 2, \alpha=\rho^{2} / 4, \lambda, \rho$ and $\beta$ are arbitrary

IV. $\gamma=1 / 2, \alpha=3 \rho^{2} / 4, \lambda=0, \rho$ and $\beta$ are arbitrary

These corroborate the results of [9] for reduction to the heat equation (5).

Now we focus on the situation when the bond pricing equation (15) is equivalent to the second Lie canonical form (9). That is, we invoke the invariant condition (10). Condition (10) reduces to 


$$
20 L_{x}+10\left[|a|^{1 / 2} L_{x}\right]_{x} \int d x /|a|^{1 / 2}+\left[|a|^{1 / 2}\left[|a|^{1 / 2} L_{x}\right]_{x}\right]_{x}\left(\int d x /|a|^{1 / 2}\right)^{2}=0(18
$$

since equation (15) is autonomous.

Two cases naturally arise in the evaluation of the invariant equation (18). This is due to the evaluation of the integral in (18). We therefore end up with two possible values for $\gamma$, viz. $\gamma=1$ and $\gamma \neq 1$.

For $\gamma=1$, condition (18) is

$$
\begin{aligned}
& 10 \rho^{2}+10 \alpha \rho^{2} x^{-2}-40 \alpha^{2} x^{-3}-10 \alpha \beta x^{-2}+10 \alpha \lambda \rho x^{-2}+5 \rho^{2} \ln x \\
& -5 \alpha \rho^{2} x^{-2} \ln x+40 \alpha^{2} x^{-3} \ln x+5 \alpha \beta x^{-2} \ln x-5 \alpha \lambda \rho x^{-2} \ln x+\frac{1}{2} \rho^{2} \ln ^{2} x \\
& +\frac{1}{2} \alpha \rho^{2} x^{-2} \ln ^{2} x-8 \alpha^{2} x^{-3} \ln ^{2} x-\frac{1}{2} \alpha \beta x^{-2} \ln ^{2} x+\frac{1}{2} \alpha \lambda \rho x^{-2} \ln ^{2} x=0 .
\end{aligned}
$$

The condition (19) implies $\rho=0$ which is not allowed. Thus we conclude that for $\gamma=1$, the PDE (15) is not reducible to the second Lie canonical form (9) by point transformation.

Now for $\gamma \neq 1$, condition (18) becomes

$$
\begin{aligned}
& 10 \rho^{2} \gamma(2 \gamma-1) x^{2 \gamma-2}+30 \lambda \rho^{3} \gamma(\gamma-1)^{3} x^{3 \gamma-4}-30 \rho^{4} \gamma^{2}(\gamma-1)^{3} x^{4 \gamma-5} \\
&+60 \rho^{4} \gamma(\gamma-1)^{3} x^{4 \gamma-5}+10 \alpha \rho^{2} \gamma(\gamma-2)(2 \gamma-3) x^{2 \gamma-4}-20 \alpha^{2} \gamma(\gamma+1) x^{-3} \\
&+10 \alpha \beta \gamma(1-2 \gamma) x^{-2}-10 \alpha \lambda \rho \gamma(\gamma-2) x^{\gamma-3}+5 \rho^{2} \gamma \frac{(2 \gamma-1)}{1-\gamma}(3 \gamma-2) x^{2 \gamma-2} \\
&+60 \lambda \rho^{3} \gamma(1-\gamma)^{3} x^{3 \gamma-4}-75 \rho^{4} \gamma^{2}(1-\gamma)^{3} x^{4 \gamma-5}+150 \rho^{4} \gamma(1-\gamma)^{3} x^{4 \gamma-5} \\
&+5 \alpha \rho^{2} \gamma \frac{(\gamma-2)}{1-\gamma}(2 \gamma-3)(3 \gamma-4) x^{2 \gamma-4}-10 \alpha^{2} \gamma \frac{(\gamma+1)}{1-\gamma}(\gamma-3) x^{-3} \\
&+5 \alpha \beta \gamma \frac{(1-2 \gamma)}{1-\gamma}(\gamma-2) x^{-2}-5 \alpha \lambda \rho \gamma \frac{(\gamma-2)}{1-\gamma}(2 \gamma-3) x^{\gamma-3} \\
&+\frac{1}{2} \rho^{2} \gamma \frac{(2 \gamma-1)}{(1-\gamma)^{2}}(3 \gamma-2)(4 \gamma-3) x^{2 \gamma-2}+30 \lambda \rho^{3} \gamma(\gamma-1)^{3} x^{3 \gamma-4} \\
&-45 \rho^{4} \gamma^{2}(\gamma-1)^{3} x^{4 \gamma-5}+90 \rho^{4} \gamma(\gamma-1)^{3} x^{4 \gamma-5} \\
&+\frac{1}{2} \alpha \rho^{2} \gamma \frac{(\gamma-2)}{(1-\gamma)^{2}}(2 \gamma-3)(3 \gamma-4)(4 \gamma-5) x^{2 \gamma-4}-2 \alpha^{2} \gamma \frac{(\gamma+1)}{(1-\gamma)^{2}}(\gamma-3)(\gamma-2) x^{-3} \\
&+\frac{1}{2} \alpha \beta \gamma \frac{(1-2 \gamma)}{(1-\gamma)^{2}}(\gamma-2)(2 \gamma-3) x^{-2}-\frac{1}{2} \alpha \lambda \rho \gamma \frac{(\gamma-2)}{(1-\gamma)^{2}}(2 \gamma-3)(3 \gamma-4) x^{\gamma-3}
\end{aligned}
$$

The analysis of the invariant condition (20) is the same as that for the heat invariant criterion (17). We straightforwardly deduce the following cases.
A. $\gamma=2, \alpha=0, \lambda, \beta$ and $\rho$ are arbitrary
B. $\gamma=3 / 2, \alpha=0, \lambda, \beta$ and $\rho$ are arbitrary
C. $\gamma=1 / 2, \alpha, \lambda, \beta$ and $\rho$ are arbitrary.

Case $\mathrm{A}$ is missing in the work [9]. For Cases $\mathrm{B}$ and $\mathrm{C}$ partial results are obtained in [9]. In fact for Cases B and C four nontrivial symmetries were only found for $\lambda=0$ in 
the work [9]. Hence for all the cases our results above are new.

It is easily seen from condition (12) of the Theorem that $\partial J / \partial t=0$ excluding when the invariant conditions (6) and (10) apply (as for these conditions we have the $\lambda$ values already). In this case and therefore for values of $\lambda, \alpha, \lambda, \beta$ and $\rho$ not covered by cases I to IV and A to C, the PDE (2.14) can be reduced to the third Lie canonical form (11) for which it admits two nontrivial symmetries. We do not have the condition (14) being satisfied for the PDE (15) as equation (15) is autonomous.

\section{A HAMILTONIAN VIEWPOINT FOR EQUATIONS OF ECONOMICS}

We draw attention to an important approach [12] to the equations of economics.

In economics, the maximum principle consists of first-order ordinary differential equations in what are called state and costate variables. Also the further requirement is that the current-value Hamiltonian be maximized with respect to the control variables.

Suppose that one maximizes

$$
\int_{0}^{T} H_{1}(t, q, u) e^{-r t} d t
$$

subject to

$$
\dot{q}=H_{2}(t, q, u),
$$

where the integrand contains the discount factor $e^{-r t}, t$ is the time, $q$ is the state variable and $u$ the control variable.

Then the current-value Hamiltonian is written as

$$
H(t, q, u, p)=H_{1}(t, q, u)+p H_{2}(t, q, u)
$$

in which $p$ is referred to as the costate variable. The necessary conditions for optimal control are [15] $\partial H / \partial u=0$ as well as

$$
\begin{aligned}
& \dot{q}=\partial H / \partial p, \\
& \dot{p}=-\partial H / \partial q+r p .
\end{aligned}
$$

We note that the system is not in the form of the canonical Hamiltonian equations due to the nonzero term $r p$ and hence one cannot take advantage of the reduction techniques involving first integrals developed in the classical literature on the subject. A new approach to deal with systems (24) for multi-variables was studied in [12].

\section{CONCLUDING REMARKS}

Many important applications of symmetry and operator approaches appear in the literature dating back to the works of Lie. Applications to mathematics of finance are recent. The main approach has been on the computation of the symmetry groups for various models of finance which we alluded to in the introduction. The invariant approach has also been invoked in a few papers. Here we showed how effective the invariant method is by applying it to a bond pricing equation taken from Sinkala et al. [9] that contained the Vasicek [13] and Cox-Ingersoll-Ross (CIR) [14] models. We found new cases of the parameters for which the bond pricing equation considered has four nontrivial symmetries and hence can be mapped to the second Lie canonical form. 
Acknowledgements-FMM thanks Mehmet Pakdemirli and the organizing committee of ICMCA2013 for their warm invitation. FMM, KSM and EM are grateful to the NRF of South Africa for research funding.

\section{REFERENCES}

1. R. K. Gazizov and N. H. Ibragimov, Lie symmetry analysis of differential equations in finance, International Journal of Nonlinear Dynamics 17 387-407, 1998.

2. J. Goard, New solutions to the bond-pricing equation via Lie's classical method, Mathematical and Computer Modelling 32, 299-313, 2000.

3. C. F. Lo and C. H. Hui, Valuation of financial derivatives with time-independent parameters: Lie-algebraic approach, Quantitative Finance 1,73-78, 2001.

4. C. A. Pooe, F. M. Mahomed and C. Wafo Soh, Fundamental solutions for zero-coupon bond pricing models, Nonlinear Dynamics 36, 69-76, 2004.

5. C. Sophocleous, J. G.O'Hara and P. G. L. Leach, Algebraic solution of the Stein-Stein model for stochastic volatility, Commun Nonlinear Sci Numer Simulat 16, 1752-1759, 2011.

6. I. K. Johnpillai and F. M. Mahomed, Singular invariant equation for the (1+1) FokkerPlanck equation, Journal of Physics A: Mathematical and General 34, 11033-11051, 2001.

7. F. M. Mahomed, Complete Invariant Characterization of Scalar Linear (1+1) Parabolic Equations, Journal of Nonlinear Mathematical Physics 15, Supplement 1, 112-123, 2008. 8. S. Lie, On integration of a class of linear partial differential equations by means of definite integrals, Archiv for Mathematik og Naturvidenskab, VI(3) (1881) 328-368, [in German], Reprinted in S. Lie, Gesammelte Abhadlundgen, 3 paper XXXV, 1881.

9. W. Sinkala, P. G. L. Leach and J. G.O'Hara, Invariance properties of a general bond-pricing equation, Journal of Differential Equations 244, 2820-2835, 2008.

10. J. R. Ruiz-Tamarit and M.Ventura-Marco, Solution to nonlinear MHDS arising from optimal growth problems, Mathematical Social Sciences 61 86-96, 2011.

11. C. Chilarescu, A closed-form solution to the transitional dynamics of the Lucas-Uzawa model, Economic Modelling 26, 135-138, 2009.

12. R. Naz, F. M. Mahomed and Chaudhry Azam, A Hamiltonian Approach to Equations of Economic Theory, preprint.

13. O. Vasicek, An equilibrium characterization of the term structure, Journal of Financial Economics 5, 177-188,1977.

14. J. C. Cox, J. E. Ingersoll and S. A. Ross, An intertemporal general equilibrium model of asset prices, Econometrica 53, 363-384,1985.

15. A. C. Chiang, Elements of dynamic optimization, McGraw Hill, 1992. 